Correspondence

Sean D. Reid

sreid@wfubmc.edu

Received 2 June 2008

Revised 22 September 2008

Accepted 23 October 2008

\section{Biofilm formation by group A Streptococcus: a role for the streptococcal regulator of virulence (Srv) and streptococcal cysteine protease (SpeB)}

\author{
Christopher D. Doern, ${ }^{1} \dagger$ Amity L. Roberts, ${ }^{1} \dagger$ Wenzhou Hong, ${ }^{1}$ \\ Jessica Nelson, ${ }^{2}$ Slawomir Lukomski, ${ }^{3,4}$ William E. Swords ${ }^{1}$ \\ and Sean D. Reid ${ }^{1}$
}
${ }^{1}$ Department of Microbiology and Immunology, Wake Forest University School of Medicine, Winston-Salem, NC 27157, USA
${ }^{2}$ Salem College, Winston-Salem, NC 27101, USA
${ }^{3}$ Department of Microbiology, Immunology and Cell Biology, West Virginia University, Morgantown, WV 26506, USA
${ }^{4}$ Mary Babb Randolph Cancer Center, School of Medicine, West Virginia University, Morgantown, WV 26506, USA

\begin{abstract}
Recently, biofilms have become a topic of interest in the study of the human pathogen group $A$ Streptococcus (GAS). In this study, we sought to learn more about the make-up of these structures and gain insight into biofilm regulation. Enzymic studies indicated that biofilm formation by GAS strain MGAS5005 required an extracellular protein and DNA component(s). Previous results indicated that inactivation of the transcriptional regulator Srv in MGAS5005 resulted in a significant decrease in virulence. Here, inactivation of Srv also resulted in a significant decrease in biofilm formation under both static and flow conditions. Given that production of the extracellular cysteine protease SpeB is increased in the srv mutant, we tested the hypothesis that increased levels of active SpeB may be responsible for the reduction in biofilm formation. Western immunoblot analysis indicated that SpeB was absent from MGAS5005 biofilms.

Complementation of MGAS5005 $\Delta$ srv restored the biofilm phenotype and eliminated the overproduction of active SpeB. Inhibition of SpeB with E64 also restored the MGAS5005 $\Delta s r v$ biofilm to wild-type levels.
\end{abstract}

\section{INTRODUCTION}

Group A Streptococcus (GAS) is responsible for a wide range of human infections, including pharyngitis and tonsillitis, skin infections, sepsis, a toxic-shock syndrome, and necrotizing fasciitis (Cunningham, 2000; Musser \& Krause, 1998; Reid et al., 2001). Until recently, there was little association of GAS and biofilms in the published literature. One of the first observations was reported by Akiyama et al. (2003) in a study examining GAS in skin infections. Confocal laser scanning microscopy of skin lesions revealed the presence of GAS microcolonies that appeared to be surrounded by a glycocalyx (Akiyama et al., 2003). A limited number of subsequent studies have demonstrated GAS biofilm formation in vitro and in vivo and microarray analyses have shown that gene transcription varies markedly in biofilms compared to planktoni-

†These authors contributed equally to this work.

Abbreviations: CV, crystal violet; GAS, group A Streptococcus. cally grown GAS (Baldassarri et al., 2006; Cho \& Caparon, 2005; Conley et al., 2003; Lembke et al., 2006; Manetti et al., 2007; Riani et al., 2007; Takemura et al., 2004). However, little is known about whether the ability to form biofilms is a characteristic of all GAS strains, what the biofilm matrix is composed of, or how this biofilm state is regulated.

In the present work, we demonstrate that a globally disseminated M1T1 serotype clone associated with invasive disease is capable of producing a structured, surfaceattached community resembling a biofilm. In addition, we demonstrate that DNA and protein are critical to the formation of this structure. Furthermore, in an effort to gain insight into the regulation of the biofilm process, we examined the effects of loss of the virulence regulator Srv on GAS biofilm formation. Srv has been shown to be required for GAS virulence and to influence GAS gene expression (Reid et al., 2004, 2006). Here we show that Srv is required for biofilm formation and that increased activity of the cysteine protease SpeB in the absence of Srv contributes to the loss of the biofilm phenotype. 


\section{METHODS}

Bacterial strains. MGAS5005 is a representative M1T1 serotype strain $\left(\right.$ speA $\left.^{+}\right)$which was isolated from a case of invasive GAS disease and has been the focus of numerous studies (reviewed by Musser \& Krause, 1998; Reid et al., 2001). The MGAS5005 isogenic srv mutant strain (MGAS5005 $\Delta s r v$ ) was generated by allelic replacement (Reid et al., 2004). MGAS5005 $\Delta s r v$ was complemented in trans [MGAS5005 $\Delta r v(\mathrm{pIA} \beta 8-s r v)]$ as previously described (Doern et al., 2008).

Adherence assay. Overnight cultures of GAS grown at $37{ }^{\circ} \mathrm{C}(5 \%$ $\mathrm{CO}_{2}$ ) in Todd-Hewitt Broth (TH) (Difco) supplemented with $0.2 \%$ yeast extract (THY) (Fisher Scientific) were harvested and used to inoculate fresh THY. Cultures were then grown to an $\mathrm{OD}_{600}$ of 0.5 . Tissue culture treated polystyrene six-well cell culture plates (Corning) were seeded with $3 \mathrm{ml}$ of culture per well. Plates were incubated for $24 \mathrm{~h}$ at $37{ }^{\circ} \mathrm{C}, 5 \% \mathrm{CO}_{2}$. Medium was removed without disturbing the biofilm and wells were washed three times with distilled $\mathrm{H}_{2} \mathrm{O}\left(\mathrm{dH}_{2} \mathrm{O}\right)$. Then $1 \mathrm{ml}$ aliquots of $0.1 \%$ crystal violet (CV) (Sigma-Aldrich) dissolved in $\mathrm{dH}_{2} \mathrm{O}$ were dispensed to each well. Surface-attached bacteria were allowed to stain for $30 \mathrm{~min}$ at room temperature and then washed three times with $\mathrm{dH}_{2} \mathrm{O}$, after which $1 \mathrm{ml}$ ethanol was added to each well to solubilize the CV. An $A_{600}$ reading (measuring the colour intensity of the solubilized CV) was recorded for each sample. If staining was too dark for spectrophotometric analysis, the sample was diluted with additional ethanol and the dilution factor applied to the resulting reading (in these instances, the value is referred to as the $A_{600}$ extrapolated, or $A_{600 \mathrm{e}}$ ).

Enzymic inhibition/disruption of biofilms. Methods were adapted from Wang et al. (2004). To test the ability to inhibit biofilm formation, $2 \mathrm{ml}$ aliquots of exponentially growing GAS cultures $\left(\mathrm{OD}_{600} 0.5\right)$ were mixed with $2 \mathrm{ml}$ of one of the following reagents (final concn): $40 \mathrm{mM} \mathrm{NaIO}_{4}$ (metaperiodate), $200 \mu \mathrm{g} \mathrm{DNase} \mathrm{I} \mathrm{ml}^{-1}$ or $1 \mathrm{mg}$ proteinase $\mathrm{K} \mathrm{ml}^{-1}$. Enzyme-treated cultures were added to sixwell tissue culture plates and incubated for $24 \mathrm{~h}$ at $37{ }^{\circ} \mathrm{C}, 5 \% \mathrm{CO}_{2}$. Wells were washed and stained with $\mathrm{CV}$ as described above. To test the ability to disrupt a pre-formed biofilm, each of the reagents described above was added to a 24-h-old biofilm and allowed to incubate for an additional $1 \mathrm{~h}$. Wells were washed and stained as before.

\section{Analysis of biofilm formation under continuous-flow condi- tions. Using a 25 gauge $1 \frac{1}{2}$ inch Precision Glide needle (Becton Dickinson), a $10 \mathrm{ml}$ aliquot of exponentially growing GAS culture $\left(\mathrm{OD}_{600}\right.$ 0.5) was inoculated into a convertible flow-cell chamber (Stovall Life Sciences). Each inoculum was incubated for $3 \mathrm{~h}$ at $37{ }^{\circ} \mathrm{C}$ without flow to allow initial attachment within the chamber. Sterile THY medium was connected to the flow chamber through the pump head via Masterflex silicon tubing (peroxide treated) L/S14 (Cole- Parmer Instruments), and flow discharge was collected in a waste container downstream of the flow-cell chamber. Flow was initiated using a Masterflex Easy-Load II peristaltic pump (Cole-Parmer Instruments) with a flow rate of $0.7 \mathrm{ml} \mathrm{min}^{-1}$ and continued for $24 \mathrm{~h}$ at $37{ }^{\circ} \mathrm{C}$.}

Confocal laser scanning microscopy of flow-cell biofilms. To visually examine the health of the biofilm, samples were subjected to live/dead staining. Samples contained within flow-cell chambers were washed with PBS. A mixture of SYTO 9 and propidium iodide (LIVE/ DEAD BacLight Bacterial Viability kit L7007; Molecular Probes) contained in $10 \mathrm{ml}$ PBS was added to the chamber. The sample was allowed to incubate at room temperature for $1 \mathrm{~h}$ with the LIVE/ DEAD stain and then washed with PBS. Samples were visualized using a Zeiss LSM510 confocal scanning laser microscope.
Scanning electron microscopy of flow-cell biofilms. Continuous flow-cell samples were fixed within the flow-cell chamber with $2.5 \%$ glutaraldehyde in PBS. After fixation for a minimum of $1 \mathrm{~h}$, samples were washed twice and subjected to dehydration, fixation, and critical-point drying for scanning electron microscopy. Flow-cell chamber slides were then trimmed, mounted, and coated with palladium. Biofilms were viewed on a Phillips SEM-515 scanning electron microscope.

\section{RESULTS}

\section{Protein and DNA are significant components of the GAS biofilm}

We first determined the level of biofilm formed by MGAS5005, a representative serotype M1T1 strain recently isolated from a case of invasive GAS disease. Population genetic analysis has indicated that M1T1 strains are among the most common causes of invasive GAS infections worldwide in most case studies (Musser \& Krause, 1998; Reid et al., 2001). Dilution of the solubilized CV was necessary as the original CV staining was too dark for spectrophotometric analysis.

We next sought to learn more about the biofilm structure by examining the components present. We hypothesized that if proteins, DNA or polysaccharides are required, then biofilms should be inhibited or disrupted by proteinase K, DNase I, or metaperiodate, respectively. Addition of proteinase $\mathrm{K}$ or DNase I at the time six-well plates were seeded significantly inhibited biofilm formation (Fig. 1a). In addition, a $1 \mathrm{~h}$ incubation with either proteinase K or DNase I disrupted an existing $24 \mathrm{~h}$ old biofilm (Fig. 1b). Treatment with metaperiodate, a compound capable of oxidizing polysaccharides (Mack et al., 1996; Maira-Litran et al., 2002; Wang et al., 2004), led to a reduction in both the formation and the stability of the biofilm, but measurable biofilm was still present (Fig. 1a, b). As a control, we performed replicate plating to test the viability of the bacteria following treatment. C.f.u. numbers recovered following treatment with proteinase $\mathrm{K}$ or DNase I were equivalent to those following treatment with PBS $\left(\sim 10^{9}\right.$ c.f.u. $\left.\mathrm{ml}^{-1}\right)$. However, we recovered $10^{6}-10^{7}$ c.f.u. $\mathrm{ml}^{-1}$ from metaperiodate-treated samples. This suggested that the decrease in biofilm observed may be due to the effects of metaperiodate on bacterial viability. Thus, the GAS biofilm requires a protein and a DNA component(s) for formation and stability. GAS polysaccharides may increase the mass of the biofilm when present, but periodate-sensitive polysaccharides do not appear to be required for GAS biofilm formation.

\section{Inactivation of the transcriptional regulator Srv results in a significant reduction in GAS biofilm formation}

The observation that extracellular protein is important in the formation of the GAS biofilm led us to consider the possible role of the transcriptional regulator Srv. Our previous studies indicated that inactivation of $s r v$ resulted 


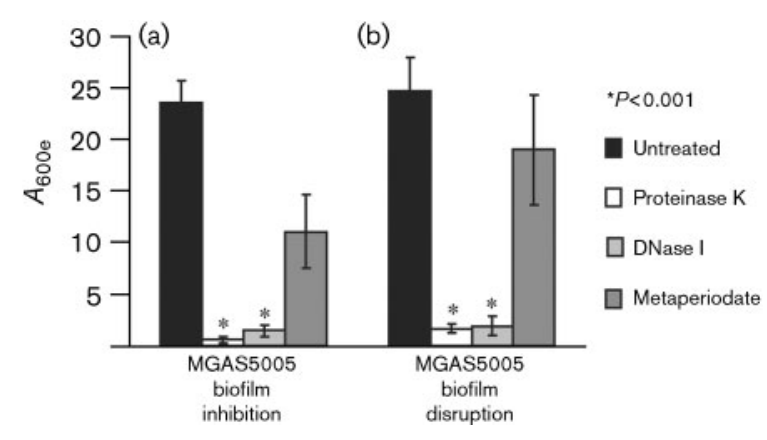

Fig. 1. Enzymic inhibition or disruption of GAS biofilm formation. (a) To test the ability of specific enzymes to inhibit biofilm formation, proteinase K, DNase I or metaperiodate was added when the six-well plates were initially seeded with MGAS5005 in the exponential phase $\left(O D_{600}\right.$ 0.5). After $24 \mathrm{~h}$ growth, strains incubated with proteinase $\mathrm{K}$ or DNase I produced significantly less, if any, biofilm. While metaperiodate curtailed biofilm formation, it did not abolish this ability. Each reported value is the mean \pm SD of at least six replicates and is adjusted by the dilution factor required to obtain a spectrophotometric reading $\left(A_{600}\right.$ extrapolated or $\left.A_{600 \mathrm{e}}\right)$. (b) To test the ability of the same enzymes to disrupt an established GAS biofilm, proteinase K, DNase I or metaperiodate was added to cultures grown in six-well plates for $24 \mathrm{~h}$. CV assays were completed after $1 \mathrm{~h}$ incubation with enzyme. Treatment with proteinase $\mathrm{K}$ or DNase I disrupted the biofilm and yielded a significantly reduced $A_{600 e}$. Treatment with metaperiodate reduced the amount of biofilm present, but was unable to completely disrupt the biofilm.

in a significant reduction in GAS virulence (Reid et al., 2004) and differential transcription of 51 genes encoding proven or putative extracellular proteins (Reid et al., 2006). The reduced expression of genes encoding extracellular proteins in the srv mutant strain led us to hypothesize that Srv may contribute to biofilm formation. Comparison of MGAS5005 and the srv isogenic mutant derivative MGAS5005 $\Delta s r v$ indicated that MGAS5005 $\Delta s r v$ produced significantly less biofilm (Fig. 2a). Complementation of $s r v$ in trans [MGAS5005 $\Delta r v(\mathrm{pIA} \beta 8$-srv)] restored biofilm production to near wild-type levels (Fig. 2a).

To determine if the results observed were due to an impaired ability of MGAS5005 ssrv to form biofilms or an early disruption of the biofilm, we followed formation of the structure over time. MGAS5005 and MGAS5005 $\Delta s r v(\mathrm{pIA} \beta 8$ $s r v)$ produced biofilm at approximately the same rate and to the same level (Fig. 2b). Maximal biofilm formation was observed at $24 \mathrm{~h}$, with levels tapering off thereafter. In contrast, CV staining of surface-attached MGAS5005 s srv was significantly less at all time points measured (Fig. 2b).

\section{Inhibition of SpeB in MGAS5005 $\Delta$ srv restores biofilm formation}

Our data suggested that Srv-mediated gene transcription was required for proper biofilm formation. This may be
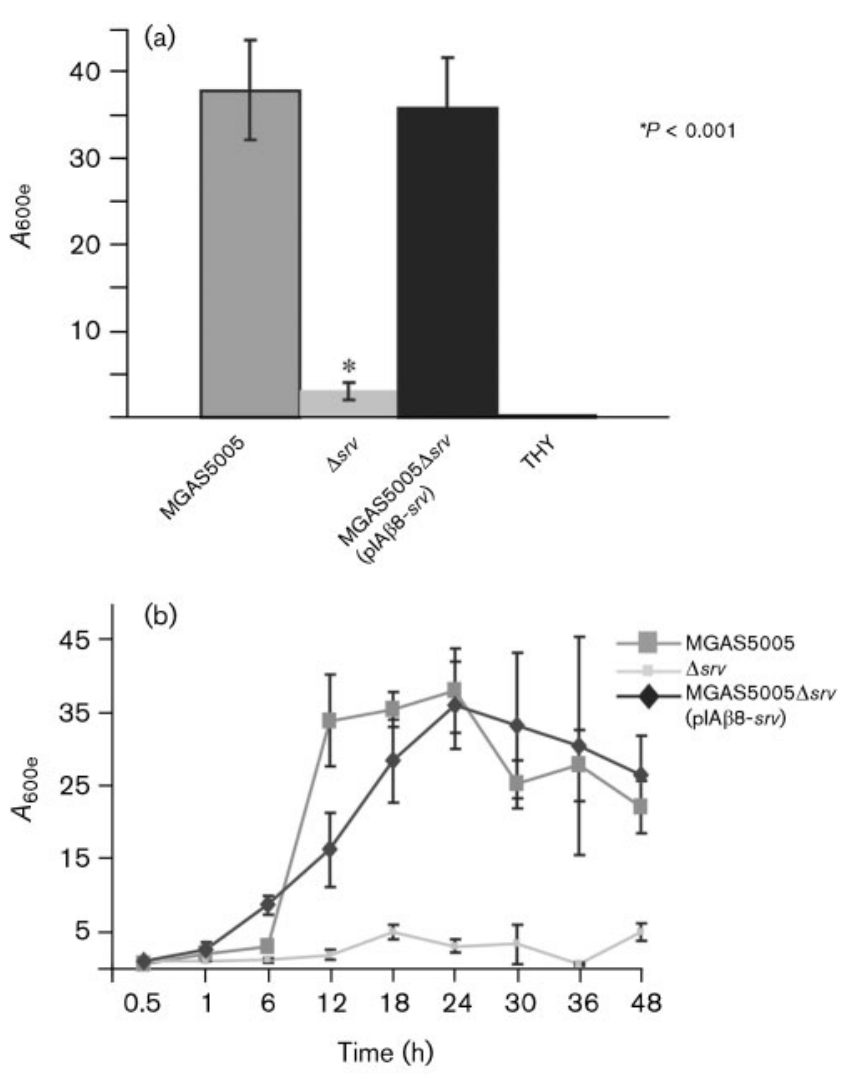

Fig. 2. The transcriptional regulator Srv is required for maximal biofilm formation by MGAS5005. Each reported value for the CV assay is an average of at least six replicates and is adjusted by the dilution factor required to obtain a spectrophotometric reading $\left(A_{600 \mathrm{e}}\right)$. (a) A comparison of the wild-type (MGAS5005), the isogenic mutant lacking $s r v(\Delta s r v)$, and the complemented strain [MGAS5005 $\Delta s r v(p I A \beta 8-s r v)]$. The $\Delta s r v$ mutant is significantly reduced in its ability to form biofilms. Complementation of $s r v$ in trans restores biofilm formation. The mean $A_{600}$ (extrapolation unnecessary) for wells containing THY alone is provided as a negative control. (b) Kinetics of GAS biofilm formation. The $A_{600 e}$ values of solubilized CV from surface-attached cells grown in sixwell polystyrene plates were assayed over time.

due to reduced transcription of genes encoding extracellular proteins required for attachment and/or aggregation, or to the effects of some other product that is directly or indirectly affected by Srv. Previously, we discovered that inactivation of $s r v$ led to increased production of the GAS cysteine protease SpeB (Reid et al., 2006). We can visualize the increased activity of SpeB in a casein agar assay, in which strains are stab inoculated into casein agar and allowed to grow at $37{ }^{\circ} \mathrm{C}$ under microaerophilic conditions. The resulting zone of translucence surrounding the stab site is indicative of SpeB caseinolytic activity. This assay demonstrated that complementation with srv in trans restored SpeB activity to wild-type levels (Fig. 3).

To test the hypothesis that an increase in SpeB may be responsible for the MGAS5005 $\Delta$ srv biofilm defect, we 


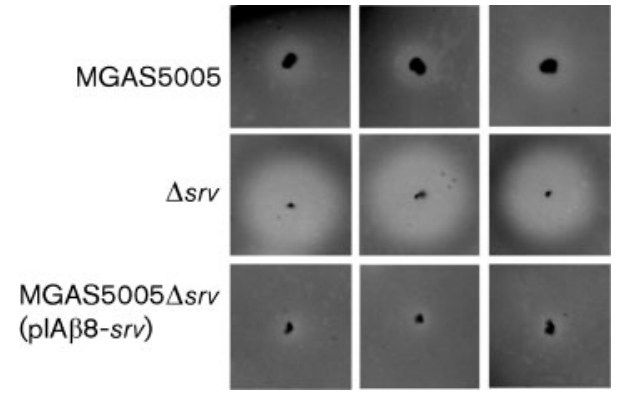

Fig. 3. Casein agar assay for SpeB proteolytic activity. MGAS5005, $\Delta s r v$ and MGAS5005 $\Delta s r v(p I A \beta 8-s r v)$ were stab inoculated into agar plates containing skim milk and incubated for $18 \mathrm{~h}$ microaerophilically. Protease activity is manifest as a zone of translucence surrounding the stab sites. There is a marked increase in the amount of SpeB proteolysis exhibited by $\Delta s r v$ compared to MGAS5005 or MGAS5005 $\Delta$ srv(pIA $\beta 8$-srv).

examined biofilm formation in the presence of an inhibitor of SpeB activity, E64. E64 is a potent and highly selective cysteine protease inhibitor that irreversibly binds to an active thiol group to form a thioether linkage. Previous groups have used concentrations of $\sim 30 \mu \mathrm{M}$ to $10 \mathrm{mM}$ E64 (Kansal et al., 2003; Vincents et al., 2004). However, in these studies the inhibitor was added to assays using GAS supernatants or purified proteins. Since we wished to add E64 to living cells and allow the culture to incubate for $24 \mathrm{~h}$, we elected to use a final concentration of $333 \mu \mathrm{M}$. Addition of E64 at the time the plates were seeded restored the ability of MGAS5005 $\Delta$ s $r v$ to form biofilms (Fig. 4a). To determine that the observed results were due to the inhibition of SpeB and not another protease, we examined the biofilm formation of a speB isogenic mutant (Lukomski et al., 1998) with and without the addition of E64 (Fig. 4a). No difference was observed in the ability of the $\Delta$ speB strain to form biofilms. Examination of the attached bacteria prior to solubilization with ethanol revealed that the CV stain was not as uniform in E64-treated MGAS5005 srv as in the treated wild-type, but there was a clear increase in attached bacteria compared to untreated MGAS5005 $\Delta r v$ (Fig. 4b).

\section{Extracellular SpeB is absent from $24 \mathrm{~h}$ GAS biofilms}

Previously, we demonstrated that SpeB was detected by Western immunoblotting in culture supernatants of MGAS5005 following $8 \mathrm{~h}$ of planktonic growth (Reid et al., 2006). By contrast, SpeB was detectable in MGAS5005 s srv supernatants after just $2 \mathrm{~h}$ of growth (Reid et al., 2006). For the experiments presented above, cultures were allowed to grow planktonically for $3 \mathrm{~h}$ prior to seeding of the six-well plates. Biofilms were then allowed to develop for $24 \mathrm{~h}$. If SpeB inhibits or disperses the GAS biofilm, then it must be present at very low levels if at all in

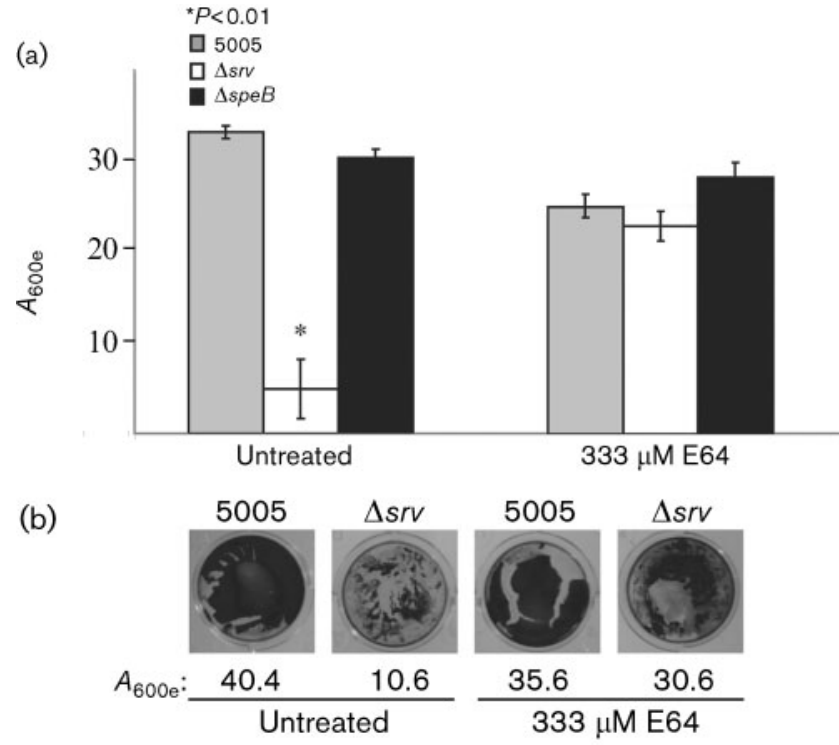

Fig. 4. Inhibition of SpeB activity restores GAS biofilm formation. (a) Graphical representation of CV assays of biofilm-forming ability in the presence and absence of the cysteine protease inhibitor E64. While addition of E64 may reduce the biofilm capacity of the wild-type slightly, biofilm formation of the $\Delta s r v$ strain is restored to comparable levels. E64 did not alter biofilm formation by the $\Delta s p e B$ isogenic mutant strain, suggesting that the alteration in biofilm phenotype is due to SpeB activity. The data are means $\pm S D$ of at least six replicates. (b) Single-well images of CV assays prior to solubilization in ethanol. After image capture, the $\mathrm{CV}$ from surface-attached cells was solubilized and the $A_{600}$ values are shown. Note the increase in stained material in the $\Delta s r v$ well in the presence of E64.

the biofilm. To test this hypothesis, GAS biofilms and their supernatants were collected, treated as a single sample (biofilm and supernatant together), and probed by Western immunoblot analysis. Abundant SpeB was detected in the sample from the biofilm-deficient MGAS5005 $\Delta s r v$ (Fig. 5). However, SpeB was undetectable in MGAS5005 samples, and only trace amounts of SpeB-zymogen (inactive) were detected in MGAS5005 $\Delta r r v$ (pIA $\beta 8$ - $s r v$ ) samples (Fig. 5). As a negative control, samples from MGAS5005 $\Delta$ speB were probed (Fig. 5) (Lukomski et al., 1998).

\section{Analysis of GAS biofilm formation using continuous flow}

Research has indicated that the growth and structures of bacterial biofilms are affected by hydrodynamic shear that may be generated by the flow of fluids over epithelial surfaces in the host (Lappin-Scott \& Bass, 2001; Purevdorj et al., 2002; Stoodley et al., 2001). While our data indicate that GAS is capable of forming dense, surface-attached communities under static conditions, we sought to determine the ability of GAS to form biofilms under continuous flow. The convertible flow-cell chamber was 


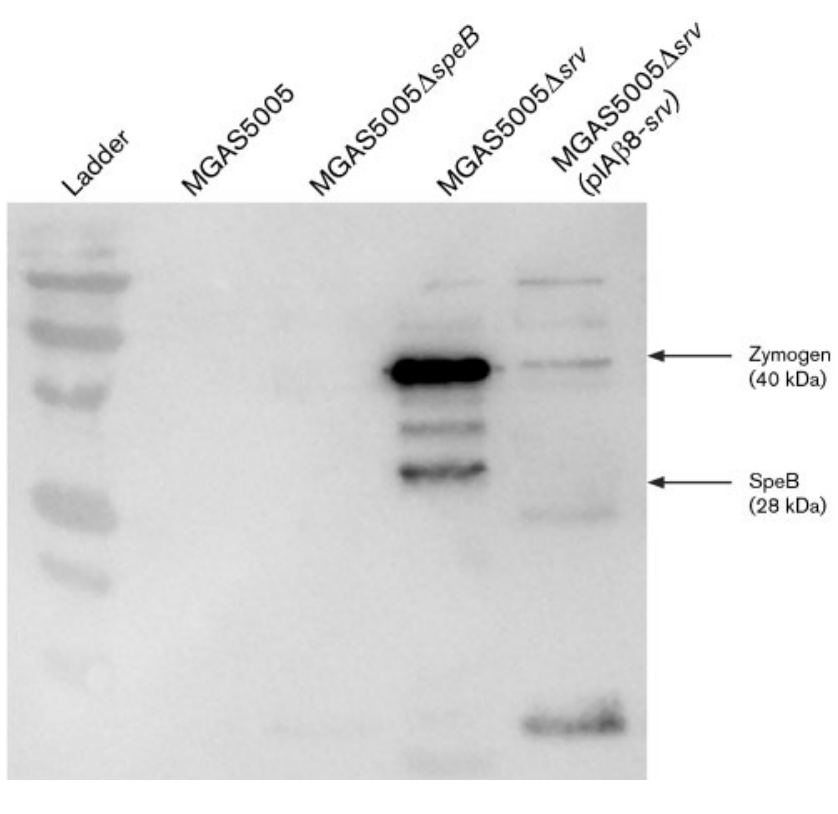

Fig. 5. Detection of SpeB in GAS biofilms. Western immunoblot analysis failed to detect the presence of SpeB in supernatants collected from MGAS5005 and MGAS5005 $\Delta$ speB after growth for $24 \mathrm{~h}$ in a biofilm state. Elevated levels of SpeB were detected in supernatants collected from MGAS5005 $\Delta s r v$. Low levels of the inactive form of SpeB (zymogen) were detected in supernatants collected from the srv-complemented strain MGAS5005 $\Delta s r v(p I A \beta 8-s r v)$.

inoculated with $10 \mathrm{ml}$ of the wild-type, the $s r v$ mutant or the complemented strain, harvested at $\mathrm{OD}_{600} 0.5$, and the bacteria were allowed to attach for $3 \mathrm{~h}$ at $37^{\circ} \mathrm{C}$. Flow of fresh THY medium was then initiated at a rate of $\sim 0.7 \mathrm{ml} \mathrm{min}^{-1}$, and the chamber was incubated for an additional $24 \mathrm{~h}$. After $24 \mathrm{~h}$ of growth, the MGAS5005 and the MGAS5005 $\Delta s r v($ pIA $\beta 8$-srv) flow chambers were completely filled with a viscous material (Fig. 6a). Plating of a sample of this material revealed GAS and no contaminants (data not shown). As observed under static conditions, MGAS5005 $\Delta s r v$ was largely unable to form a biofilm (Fig. 6a). Small pockets of attachment were observed for MGAS5005 $\Delta s r v$, but these pockets were washed away when the chamber was flushed with PBS in preparation for microscopic analyses. Taken together, these data and our analysis of static biofilms over time (Fig. 2b) suggested that MGAS5005 $\Delta s r v$ was deficient in attachment.

To determine the viability of cells within the biofilm, the MGAS5005 within the flow-cell was stained with a commercial reagent (LIVE/DEAD, Molecular Probes) and visualized by confocal microscopy (Fig. 6b). By this method, living cells with intact membranes are stained green with SYTO 9 while dead cells are stained red with propidium iodide. Colocalization of both signals gives a yellow colour. After $24 \mathrm{~h}$, the majority of cells in the biofilm were alive. Fifty consecutive micrographs of an $X-Y$ plane indicated that the biofilm was approximately $80 \mu \mathrm{m}$ thick. Vertical stacked $Z$ images (margins of Fig. 6b) showed living cells dispersed throughout the macroscopic structure.

Electron microscopy revealed a densely packed population of MGAS5005 that appeared to be encased in an extracellular matrix (Fig. 6c, d). An angled image of the biofilm removed from the corner of the flow chamber revealed the three-dimensional nature of the structure (a)

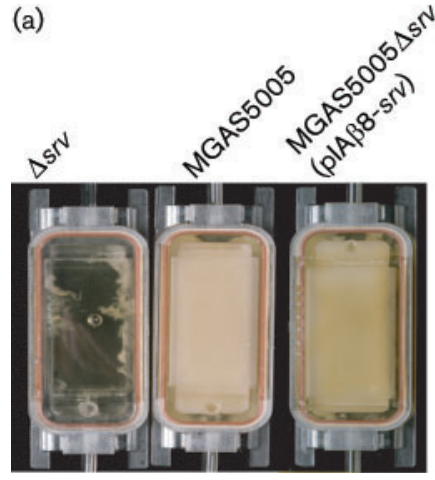

(b)

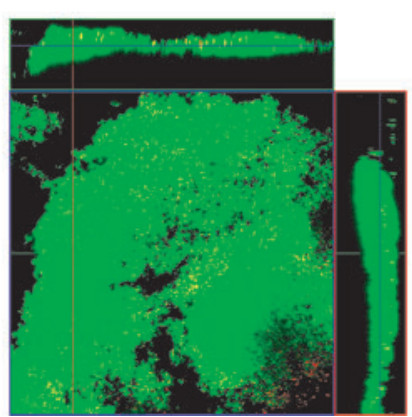

(c)

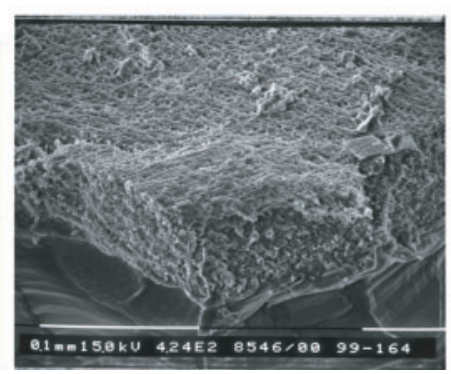

(d)

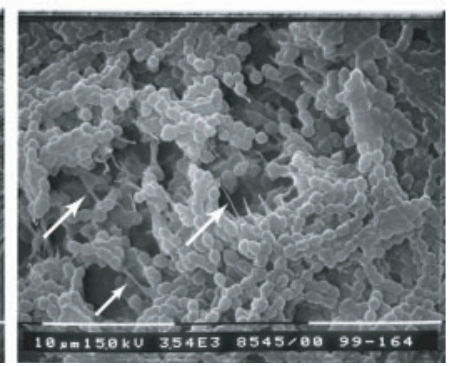

Fig. 6. Continuous-flow system for study of GAS biofilm formation. (a) Inactivation of srv eliminates the ability of GAS to form biofilms under continuous-flow conditions. Complementation of srv in trans [MGAS5005 $\Delta s r v(p I A \beta 8$-srv)] restores the biofilm phenotype. (b) Confocal microscopy of a $24 \mathrm{~h}$ continuous-flow biofilm. The image is an $X-Y$ micrograph of MGAS5005 biofilm stained with LIVE/DEAD reagent. Cells with intact membranes (living) are stained green while cells with disrupted membranes are stained red. Areas of overlap appear yellow. Fifty $X-Y$ plane micrographs indicate that the biofilm is approximately $80 \mu \mathrm{m}$ thick. Vertical stacked $Z$ images (margins) show living cells dispersed throughout the macroscopic structure. (c, d) Scanning electron microscopy of a 24 h continuous-flow biofilm; (c) shows the thickness of the biofilm formed within the chamber. At high magnification (d), chains of GAS can be clearly seen. Note the matrix material that appears to cover the bacterial chains. White arrows indicate the presence of fibrous strands. 
(Fig. 6c). Higher magnification allowed the visualization of chains of bacteria which appeared to be coated in matrix (Fig. 6d). Fibrous strands could be seen running within the matrix (Fig. 6d, white arrows). Some of these appeared to be sheared, probably during the fixation process.

\section{DISCUSSION}

In a recent study, Baldassarri et al. (2006) examined a total of 289 GAS isolates from different clinical sources and demonstrated that $90 \%$ of those strains were capable of forming biofilms. In this investigation, we sought to learn more about the make-up of these structures and gain insight into biofilm regulation. Enzymic treatment of GAS biofilms demonstrated a requirement for DNA and protein in the structure, but suggested only a passive role for carbohydrate. This is in contrast to numerous systems, for example Pseudomonas aeruginosa, for which several genetically encoded polysaccharides have been shown to be required (Donlan, 2001; Donlan \& Costerton, 2002; Hall-Stoodley et al., 2004; Ryder et al., 2007). Cho \& Caparon (2005) found that the GAS hyaluronic acid capsule (HasA) was not required for biofilm formation in a static system (CV assay), but that following initial attachment, the hasA mutant was unable to aggregate under flow conditions. Given the volume of enzyme that would be required, we were unable to test inhibition or disruption under flow conditions. Thus, hyaluronic acid does not appear necessary for initial biofilm attachment, but it may be needed for aggregation and biofilm maturation. Alternatively, differences between the serotype M1 strain used here and the Cho M5 strain may account for the observed differences. Further assessment in vivo will address these possibilities.

Increasing evidence suggests not only that DNA is a major component of bacterial biofilms, but that genetically encoded systems controlling programmed cell death provide a mechanism for the release of bacterial DNA (reviewed by Bayles, 2007). Therefore, it is not surprising that we demonstrated a role for DNA in GAS biofilms. However, the process by which GAS releases DNA is not yet known. GAS does not appear to encode cid/lrg homologues which control programmed cell death in other bacterial species, although the presence of additional or diverged genes encoding holins cannot be ruled out.

DNA, or more appropriately the lack of extracellular DNA, has been linked to severe GAS infection. Evidence has been presented that MGAS5005 has a mutation within the GAS control of virulence operon $(\operatorname{cov} R S / c s r R S)$ which limits or abolishes $s p e B$ expression. This is hypothesized to allow the production of a GAS extracellular DNase (Sda1), a protein that would normally be degraded by SpeB (Buchanan et al., 2006; Sumby et al., 2005; Walker et al., 2007). According to this model, Sdal facilitates the escape from neutrophil extracellular traps (NETs) by degrading the associated neutrophil DNA (Brinkmann et al., 2004; Walker et al.,
2007). The infecting strain is then free to disseminate and potentially cause severe invasive disease (Sumby et al., 2006; Walker et al., 2007). Our data indicate that DNA is required for MGAS5005 biofilm formation in vitro. This suggests that production of SdaI is not solely dependent on the absence of SpeB, but that it requires a signal encountered in vivo. This idea is in concert with the data of Sumby et al. (2006), who found naturally occurring examples of the covS mutation following passage in a mouse model.

It is of note that our data also indicate that inactivation of $s r v$ in MGAS5005 compensates for the covS mutation and restores $\mathrm{SpeB}$ production in this strain. In addition, our data indicate that there is a necessary protein component for initial adherence and/or aggregation leading to biofilm maturation. We hypothesized that the high levels of SpeB may be responsible for the biofilm-deficient phenotype of the srv mutant. Addition of the cysteine protease inhibitor E64 restored the ability of MGAS5005 $\Delta s r v$ to form biofilms, suggesting that SpeB degrades GAS proteins needed for establishment of the biofilm (E64-treated MGAS5005 $\Delta$ speB produced wild-type levels of biofilm). This leads to the hypothesis that the timed production of SpeB might contribute to GAS biofilm dispersion. Under this model, GAS begins in a biofilm state. In reaction to an unknown stimulus, an alteration in Srv-mediated control occurs and results in the direct or indirect increase of SpeB production and/or secretion. SpeB degrades GAS and host proteins integral to the biofilm. This alone may disperse the biofilm or, as put forth by others, the increased activity of and resulting damage from SpeB is perceived as a signal (in addition to host signals) which drives selection for mutations in covS (Sumby et al., 2006; Walker et al., 2007). Inactive CovS leads to the repression of speB, induction of sdaI, and the degradation of host and bacterial DNA (which may be more accessible due to the prior cleavage of proteins by SpeB). GAS bacteria are now free to disperse and potentially adopt an invasive phenotype (Sumby et al., 2006; Walker et al., 2007). While this model is preliminary, we believe it is testable. Clearly, further study of GAS biofilm formation will provide new insights into the pathogenesis of GAS.

\section{ACKNOWLEDGEMENTS}

This project was supported by Public Health Service Grant R01AI063453 from the National Institutes of Health to S. D. R. We thank D. J. Wozniak for critical reading of the manuscript as well as Robert C. Holder for technical assistance.

\section{REFERENCES}

Akiyama, H., Morizane, S., Yamasaki, O., Oono, T. \& Iwatsuki, K. (2003). Assessment of Streptococcus pyogenes microcolony formation in infected skin by confocal laser scanning microscopy. J Dermatol Sci 32, 193-199.

Baldassarri, L., Creti, R., Recchia, S., Imperi, M., Facinelli, B., Giovanetti, E., Pataracchia, M., Alfarone, G. \& Orefici, G. (2006). Therapeutic failures of antibiotics used to treat macrolide-susceptible 
Streptococcus pyogenes infections may be due to biofilm formation. $J$ Clin Microbiol 44, 2721-2727.

Bayles, K. W. (2007). The biological role of death and lysis in biofilm development. Nat Rev Microbiol 5, 721-726.

Brinkmann, V., Reichard, U., Goosmann, C., Fauler, B., Uhlemann, Y., Weiss, D. S., Weinrauch, Y. \& Zychlinsky, A. (2004). Neutrophil extracellular traps kill bacteria. Science 303, 1532-1535.

Buchanan, J. T., Simpson, A. J., Aziz, R. K., Liu, G. Y., Kristian, S. A., Kotb, M., Feramisco, J. \& Nizet, V. (2006). DNase expression allows the pathogen group A Streptococcus to escape killing in neutrophil extracellular traps. Curr Biol 16, 396-400.

Cho, K. H. \& Caparon, M. G. (2005). Patterns of virulence gene expression differ between biofilm and tissue communities of Streptococcus pyogenes. Mol Microbiol 57, 1545-1556.

Conley, J., Olson, M. E., Cook, L. S., Ceri, H., Phan, V. \& Davies, H. D. (2003). Biofilm formation by group A streptococci: is there a relationship with treatment failure? J Clin Microbiol 41, 4043-4048.

Cunningham, M. W. (2000). Pathogenesis of group A streptococcal infections. Clin Microbiol Rev 13, 470-511.

Doern, C. D., Holder, R. C. \& Reid, S. D. (2008). Point mutations within the streptococcal regulator of virulence (Srv) alter proteinDNA interactions and Srv function. Microbiology 154, 1998-2007.

Donlan, R. M. (2001). Biofilm formation: a clinically relevant microbiological process. Clin Infect Dis 33, 1387-1392.

Donlan, R. M. \& Costerton, J. W. (2002). Biofilms: survival mechanisms of clinically relevant microorganisms. Clin Microbiol Rev 15, 167-193.

Hall-Stoodley, L., Costerton, J. W. \& Stoodley, P. (2004). Bacterial biofilms: from the natural environment to infectious diseases. Nat Rev Microbiol 2, 95-108.

Kansal, R. G., Nizet, V., Jeng, A., Chuang, W. J. \& Kotb, M. (2003). Selective modulation of superantigen-induced responses by streptococcal cysteine protease. J Infect Dis 187, 398-407.

Lappin-Scott, H. M. \& Bass, C. (2001). Biofilm formation: attachment, growth, and detachment of microbes from surfaces. Am J Infect Control 29, 250-251.

Lembke, C., Podbielski, A., Hidalgo-Grass, C., Jonas, L., Hanski, E. \& Kreikemeyer, B. (2006). Characterization of biofilm formation by clinically relevant serotypes of group A streptococci. Appl Environ Microbiol 72, 2864-2875.

Lukomski, S., Burns, E. H., Jr, Wyde, P. R., Podbielski, A., Rurangirwa, J., Moore-Poveda, D. K. \& Musser, J. M. (1998). Genetic inactivation of an extracellular cysteine protease (SpeB) expressed by Streptococcus pyogenes decreases resistance to phagocytosis and dissemination to organs. Infect Immun 66, 771-776.

Mack, D., Fischer, W., Krokotsch, A., Leopold, K., Hartmann, R., Egge, H. \& Laufs, R. (1996). The intercellular adhesin involved in biofilm accumulation of Staphylococcus epidermidis is a linear $\beta-1,6-$ linked glucosaminoglycan: purification and structural analysis. $J$ Bacteriol 178, 175-183.

Maira-Litran, T., Kropec, A., Abeygunawardana, C., Joyce, J., Mark, G., III, Goldmann, D. A. \& Pier, G. B. (2002). Immunochemical properties of the staphylococcal poly- $N$-acetylglucosamine surface polysaccharide. Infect Immun 70, 4433-4440.

Manetti, A. G., Zingaretti, C., Falugi, F., Capo, S., Bombaci, M., Bagnoli, F., Gambellini, G., Bensi, G., Mora, M. \& other authors (2007). Streptococcus pyogenes pili promote pharyngeal cell adhesion and biofilm formation. Mol Microbiol 64, 968-983.
Musser, J. M. \& Krause, R. M. (1998). The revival of group A streptococcal diseases, with a commentary on staphylococcal toxic shock syndrome. In Emerging Infections, pp. 185-218. Edited by R. M. Krause. Academic Press.

Purevdorj, B., Costerton, J. W. \& Stoodley, P. (2002). Influence of hydrodynamics and cell signaling on the structure and behavior of Pseudomonas aeruginosa biofilms. Appl Environ Microbiol 68, 44574464.

Reid, S. D., Hoe, N. P., Smoot, L. M. \& Musser, J. M. (2001). Group A Streptococcus: allelic variation, population genetics, and hostpathogen interactions. J Clin Invest 107, 393-399.

Reid, S. D., Montgomery, A. G. \& Musser, J. M. (2004). Identification of srv, a PrfA-like regulator of group A Streptococcus that influences virulence. Infect Immun 72, 1799-1803.

Reid, S. D., Chaussee, M. S., Doern, C. D., Chaussee, M. A., Montgomery, A. G., Sturdevant, D. E. \& Musser, J. M. (2006). Inactivation of the group A Streptococcus regulator srv results in chromosome wide reduction of transcript levels, and changes in extracellular levels of Sic and SpeB. FEMS Immunol Med Microbiol 48, 283-292.

Riani, C., Standar, K., Srimuang, S., Lembke, C., Kreikemeyer, B. \& Podbielski, A. (2007). Transcriptome analyses extend understanding of Streptococcus pyogenes regulatory mechanisms and behavior toward immunomodulatory substances. Int J Med Microbiol 297, 513-523.

Ryder, C., Byrd, M. \& Wozniak, D. J. (2007). Role of polysaccharides in Pseudomonas aeruginosa biofilm development. Curr Opin Microbiol 10, 644-648.

Stoodley, P., Jacobsen, A., Dunsmore, B. C., Purevdorj, B., Wilson, S., Lappin-Scott, H. M. \& Costerton, J. W. (2001). The influence of fluid shear and AICI3 on the material properties of Pseudomonas aeruginosa PAO1 and Desulfovibrio sp. EX265 biofilms. Water Sci Technol 43, 113120.

Sumby, P., Barbian, K. D., Gardner, D. J., Whitney, A. R., Welty, D. M., Long, R. D., Bailey, J. R., Parnell, M. J., Hoe, N. P. \& other authors (2005). Extracellular deoxyribonuclease made by group A Streptococcus assists pathogenesis by enhancing evasion of the innate immune response. Proc Natl Acad Sci U S A 102, 1679-1684.

Sumby, P., Whitney, A. R., Graviss, E. A., Deleo, F. R. \& Musser, J. M. (2006). Genome-wide analysis of group A streptococci reveals a mutation that modulates global phenotype and disease specificity. PLoS Pathog 2, e5.

Takemura, N., Noiri, Y., Ehara, A., Kawahara, T., Noguchi, N. \& Ebisu, S. (2004). Single species biofilm-forming ability of root canal isolates on gutta-percha points. Eur J Oral Sci 112, 523-529.

Vincents, B., von Pawel-Rammingen, U., Björck, L. \& Abrahamson, M. (2004). Enzymatic characterization of the streptococcal endopeptidase, IdeS, reveals that it is a cysteine protease with strict specificity for IgG cleavage due to exosite binding. Biochemistry 43, 1554015549.

Walker, M. J., Hollands, A., Sanderson-Smith, M. L., Cole, J. N., Kirk, J. K., Henningham, A., McArthur, J. D., Dinkla, K., Aziz, R. K. \& other authors (2007). DNase Sdal provides selection pressure for a switch to invasive group A streptococcal infection. Nat Med 13, 981-985.

Wang, X., Preston, J. F., III \& Romeo, T. (2004). The $p g a A B C D$ locus of Escherichia coli promotes the synthesis of a polysaccharide adhesin required for biofilm formation. J Bacteriol 186, 2724-2734.

Edited by: T. J. Mitchell 